\title{
A Novel Design of Pilot Aided Channel Estimation for MIMO-CDMA System
}

\author{
Khalid Mahmood \\ University of Technology Nowshera, Pakistan
}

\begin{abstract}
In order to estimate a fading channel characteristics, a pilot signal is propogated with traffic channel. Fading channel parameter estimation is of paramount importance as it may be utilized to design different equalization techniques. It may also be utilized to allocate weights of rake receiver to sturdiest multipaths as well as coherent reception and weighted combination of multipath constituents of wireless communication systems. In this paper, a pilot aided channel estimating technique for MIMO-CDMA systems is presented. This technique utilizes minimum mean squared error estimation of corrupted information in a flat fading channel along with noise. Simulation results predicts theoretical predictions are strongly validated for different values of SNR and users.
\end{abstract}

Keywords-Channel estimation; MIMO-CDMA; channel estimator; MMSE; Rayleigh fading channel; SNR

\section{INTRODUCTION}

Design of an efficient receiver in code division multiple access (CDMA) system is perplexing as output (received) information carrying signal is compromised by Gaussian noise corrupted, interference and random nature of a flat fading channel.

Fading channel parameter estimation is of paramount importance as it may be utilized to design linear and decision feedback equalizers. Moreover fading channel estimating techniques may also be utilized in employing well-known maximum ratio combining (MRC) method [1]. Detecting multipath flat fading channel parameters could be utilized to assign the weights of rake receiver to strong multipath(s) for a DSCDMA system [1]. Pilot channel based estimating technique was utilized in IS-95 forward link [2]. It is used as for 3rd generation (3G) system on forward as well as converse link, respectively [3].

A flat fading channel estimating technique is the one, where we utilize a fixed low pass filter whose cutoff frequency is made equal to maximum Doppler frequency (MDF) [4]. Though this technique is easier to design, but its performance degenerates when its cutoff frequency strays from MDF. Optimal filters such as Wiener and Kalman were employed in [5], [6], [7], but designing them is extremely difficult due to channel parameter statistics requirement. Pilot-aided Rayleigh fading channel estimating technique utilizing minimum mean squared error (MMSE) estimator for single-input single-output (SISO) CDMA systems is developed in [8]. In our paper, we have designed a channel estimation technique for MIMOCDMA wireless system which utilizes MMSE estimation of the impaired signal in the presence of Rayleigh fading channel and Gaussian noise (AWGN). To the best of our knowledge, multiple access interference (MAI) is simply treated as additive noise [9]. But in this research work, MMSE estimation of

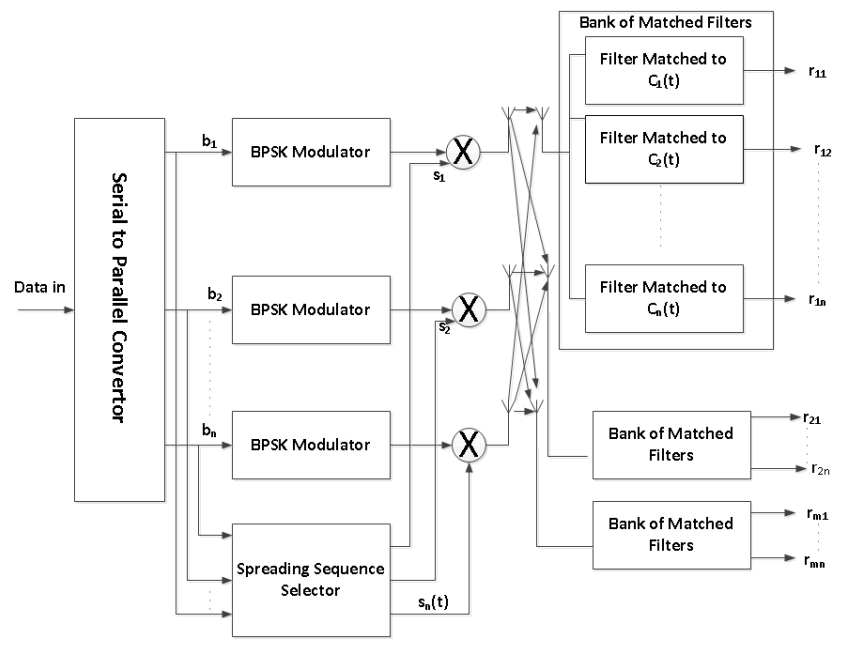

Fig. 1. System Model.

channel parameters is formed in presence MAI and noise without considering MAI as Gaussian. A unified approach of evaluating channel estimation for Rayleigh fading is also performed in this paper.

\section{SYSTEM MOdel}

CDMA transmitter model of a wireless network having $M$ transmit and $N$ receivers is shown in Fig. 1.

We are considering a Rayleigh fading channel having complex impulse response between the $n^{\text {th }}$ transmitter and $m^{t h}$ receiver for the $l^{\text {th }}$ symbol is

$$
H_{m n}^{l}(t)=h_{m n}^{l} e^{j \phi_{l}} \delta(t)
$$

Where,

$h_{m n}^{l}$ is the impulse response

$\phi_{n}$ is phase of Rayleigh fading channel for $l^{\text {th }}$ symbol.

as

The pdf of $h_{m n}^{l}$ for the Rayleigh fading channel is given

$$
\begin{aligned}
f_{h_{m n}^{l}}(x) & =\frac{u x}{\sigma_{h}^{2}} \exp \left(-\frac{u^{2}}{2 \sigma_{h}^{2}}\right), \quad \text { for } u \\
& >0
\end{aligned}
$$

Where,

$\sigma_{h}^{2}$ represents Rayleigh fading channel variance 
$m^{\text {th }}$ receiver observes the following:

$$
\begin{aligned}
r_{m}(t)= & \sum_{n=1}^{N} \sum_{l=-\infty}^{\infty} \sum_{p=1}^{P} A^{p} b_{n}^{l, p} s_{n}^{l, p}(t) h_{m n}^{l} \\
& +\nu_{m}(t), \quad m=1,2,3 \ldots M
\end{aligned}
$$

Where,

$P$ represents number of users,

$s_{n}^{l, p}(t)$ represents rectangular signature waveform with random signature sequence of the $p^{t h}$ user,

$T_{b}$ and $T_{c}$ represents bit period and chip interval,

$\left\{b_{n}^{l, p}\right\}$ represents input bit sequence of $p^{t h}$ user,

$A^{p}$ represents transmitted amplitude of $p^{t h}$ user, $\sigma_{\nu}^{2}$

$\nu_{m}$ represents Gaussian noise with zero mean and variance

Cross correlation $(\mathrm{CC})$ between signature sequences of $j^{\text {th }}$ and $p^{t h}$ users is

$$
\begin{aligned}
\rho_{l}^{p, j} & =\int_{(l-1) T_{b}}^{l T_{b}} s_{n}^{p}(t) s_{n}^{j}(t) d t \\
& =\sum_{i=1}^{N_{c}} c_{l, i}^{p} c_{l, i}^{j}
\end{aligned}
$$

$k^{t h}\left\{c_{l, i}^{p}\right\}$ is assumed to be normalized spreading sequence of

Receiving side is comprised of matched filter and is matched to a signature waveform (signal) of desired user. We are assuming that user 1 is the desired one. So matched filter's output may be set up as

$$
\begin{aligned}
y_{m}^{l} & =\int_{(l-1) T_{b}}^{l T_{b}} r_{m}(t) s_{m}^{l, 1}(t) d t \\
& =\sum_{n=1}^{N} A^{1} b_{n}^{l, 1} h_{m n}^{l}+\kappa+\nu_{m}, \quad m=1,2,3 \ldots M,
\end{aligned}
$$

Where,

$\kappa$ is MAI at the $m^{t h}$ receiver in presence of flat fading Rayleigh channel. It may be set up as

$$
\begin{aligned}
\kappa & =\sum_{n=1}^{N} \sum_{k=2}^{P} A^{p} b_{n}^{l, p} \rho_{n}^{k, 1} h_{m n}^{l}, \quad m \\
& =1,2,3 \ldots M
\end{aligned}
$$

It may also be written as

$$
\begin{aligned}
\kappa & =\sum_{n=1}^{N} Q_{m n}^{l, p} h_{m n}^{l}, \quad m \\
& =1,2,3 \ldots M
\end{aligned}
$$

The term, $Q^{l, p}=\sum_{p=2}^{P} A^{k} b^{l, p} \rho^{p, 1}$ exhibits the Gaussian behavior [10], $Q^{l, p} \sim \mathcal{N}\left(0, \sigma_{Q}^{2}\right)$, and $\sum_{n=1}^{N} A^{1} b_{n}^{l, 1} h_{m n}^{l}$ represent required signal. It may be expressed as

$$
\sum_{n=1}^{N} A^{1} b_{n}^{l, 1} h_{m n}^{l}=A^{1} b^{l, 1} \sum_{n=1}^{N} h_{m n}^{l}
$$

$\alpha^{l}=\sum_{n=1}^{N} h_{m n}^{l}$ is being used as sum of Rayleigh fading channel and considering the fact that equal power is transmitted by all users, we are defining

$$
x \triangleq b^{1} \alpha
$$

We are dropping the time index. Equation (5) can be written as

$$
y_{m}=x+\kappa
$$

Pdf of MAI plus noise as derived in [11] is

$$
f_{\kappa}(\kappa)=\frac{1}{2 \sqrt{\pi}} \sum_{n=1}^{N} \mathcal{A} \Gamma\left(\frac{1}{2}, \mathcal{B} ; \frac{\kappa^{2}}{\mathcal{C}}\right)
$$

Where,

$$
\begin{aligned}
& \mathcal{A}=\frac{C_{n}}{\sigma_{U_{n}}} \exp (\mathcal{B}), \\
& \mathcal{B}=\frac{\sigma_{\eta}^{2}}{2 \sigma_{U_{n}}^{2}}, \\
& \mathcal{C}=4 \sigma_{U_{n}}^{2} \\
& \sigma_{U_{n}}^{2}=\sigma_{I}^{2} \sigma_{\alpha}^{2} \text { is an MAI variance in MIMO-CDMA systems }
\end{aligned}
$$
[12]

\section{MMSE ESTIMATION OF MIMO-CDMA FLAT FADING CHANNEL}

MMSE estimating method technique is well known in literature for estimation in the presence of AWGN. In this research work, this technique is extended to include MAI variance in estimating Rayleigh channel for MIMO-CDMA systems. MSE cost function of MMSE estimation is given as

$$
J=E\left[(u-\hat{u})^{2}\right]
$$

Where,

$\hat{u}$ is the estimate of $u$

A cost function is defined as

$$
\begin{gathered}
\hat{u}(v)=E[u \\
\mid v] \\
=\int_{-\infty}^{\infty} u p(u \\
\mid v) d u
\end{gathered}
$$

Estimation of $\hat{\alpha}$ is obtained from (9)

$$
\hat{\alpha}=\frac{\hat{u}}{b^{1}}
$$


In order to determine $\hat{u}$, conditional pdf $p_{u \mid v}(u \mid v)$ is needed, which is given as

$$
p_{u \mid v}(u \mid v)=\frac{p_{v \mid u}(v \mid u) p_{u}(u)}{p(v)}
$$

in order to utilize (15), $p_{u}(u)$ should be evaluated first. As $u$ is product of two random variables $c^{1}$ and $\alpha$, so its pdf may be calculated by using random variable transformation

$$
p_{u}(u)=\int_{0}^{\infty} \frac{1}{\lambda} p_{\alpha}(\lambda) p_{c}\left(\frac{u}{\lambda}\right) d \lambda
$$

Where, $p_{c}(c)=0.5[\delta(c+1)+\delta(c-1)]$ and $p_{\alpha}(\alpha)$ is the pdf of sum of Rayleigh fading random variables. pdf of the sum of Rayleigh fading random variables is given as [13]

$$
\begin{aligned}
p_{S A A}(t) & =\frac{t^{2 N-1} e^{-\frac{t^{2}}{2 b}}}{2^{N-1} b^{N}(N-1) !} \\
b & =\frac{\sigma^{2}}{N}[(2 N-1) ! !]^{1 / N}
\end{aligned}
$$

Where,

$(2 N-1) ! !=(2 N-1)(2 N-3) \cdots 3.1$ and $t=u / \sqrt{N}$ are assumed to be normalized argument.

By applying transformation technique to (17), the pdf is written as

$$
\begin{aligned}
p_{\alpha}(\alpha) & =\frac{1}{\sqrt{N}} \frac{\left(\frac{\alpha}{\sqrt{N}}\right)^{2 N-1} \exp \left(-\frac{\alpha^{2}}{2 N c}\right)}{2^{N-1} c^{N}(N-1) !}, \\
c & =\frac{\sigma^{2}}{N}[(2 N-1) ! !]^{1 / N}
\end{aligned}
$$

Pdfs in (16) may be expressed as

$$
p_{c}\left(\frac{u}{\lambda}\right)=0.5\left[\delta\left(\frac{u}{\lambda}+1\right)+\delta\left(\frac{u}{\lambda}-1\right)\right]
$$

and

$$
\begin{aligned}
p_{\alpha}(\lambda) & =\frac{1}{\sqrt{N}} \frac{\left(\frac{\lambda}{\sqrt{N}}\right)^{2 N-1} \exp \left(-\frac{\lambda^{2}}{2 N c}\right)}{2^{N-1} c^{N}(N-1) !} \\
c & =\frac{\sigma^{2}}{N}[(2 N-1) ! !]^{1 / N}
\end{aligned}
$$

Now we can express (16) as

$$
\begin{aligned}
p_{u}(u)= & \frac{\mathcal{G}}{c^{N}} \int_{0}^{\infty} \lambda^{2 N-2} \exp \left(-\frac{\lambda^{2}}{2 N c}\right) \\
& \times\left[\delta\left(\frac{u}{\lambda}+1\right)+\delta\left(\frac{u}{\lambda}-1\right)\right] d \lambda
\end{aligned}
$$

Where,

$$
\mathcal{G}=\left(\frac{1}{\sqrt{N}}\right)^{2 N}\left(\frac{1}{2}\right)^{N} \frac{1}{(N-1) !} x^{2 N-1} .
$$

Integral in (21) is calculated as

$$
p_{x}(x)=\frac{\mathcal{G}}{b^{N}} u^{2 N-1} \exp \left(-\frac{u^{2}}{2 N b}\right)
$$

Pdf of $p_{y \mid x}(v \mid u)$ is calculated by (11) to obtain

$$
p_{v \mid u}(v \mid u)=\frac{1}{2 \sqrt{\pi}} \sum_{n=1}^{N} \mathcal{A} \Gamma\left(\frac{1}{2}, \mathcal{B} ; \frac{(v-u)^{2}}{\mathcal{C}}\right)
$$

Since $\int_{-\infty}^{\infty} p_{u \mid v}(u \mid v) d u=1, p(v)$ is set up as

$$
\begin{gathered}
p(v)=\int_{-\infty}^{\infty} p_{v \mid u}(v \\
\mid u) p_{u}(u) d u
\end{gathered}
$$

Putting in the terms from (22) and (23), we found

$$
p(v)=\frac{1}{2 \sqrt{\pi}} \frac{\mathcal{G}}{b^{N}} \sum_{n=1}^{N} \mathcal{A} T_{n(1)}
$$

where $T_{n(1)}$ is

$$
T_{n(1)}=\int_{-\infty}^{\infty} \Gamma\left(\frac{1}{2}, \mathcal{B} ; \frac{(v-u)^{2}}{\mathcal{C}}\right) \exp \left(-\frac{u^{2}}{2 N b}\right) d u
$$

By Substituting pdfs of $p(v)$ and $p_{v \mid u}(v \mid u)$ in (15) and utilizing (13), $\hat{u}$ estimate is provided as

$$
\hat{u}=\sum_{n=1}^{N} \frac{T_{n(2)}}{T_{n(1)}}
$$

Where, $T_{n(2)}$ is given by

$$
T_{n(2)}=\int_{-\infty}^{\infty} u^{2 N} \Gamma\left(\frac{1}{2}, \mathcal{B} ; \frac{(v-u)^{2}}{\mathcal{C}}\right) \exp \left(-\frac{u^{2}}{2 N c}\right) d u
$$

Since we could not find any closed form solution for the integrals in (26) and (28), we have solved them numerically.

\section{Simulation Results AND Discussion}

We are using following simulation setup:

- Synchronous MIMO-CDMA system with random signature sequence having length 30 .

- $\quad$ Flat Rayleigh fading channel.

- Received signal having noise which is AWGN.

- 5 and 10 users, respectively.

- $2 \times 2$ MIMO system. 


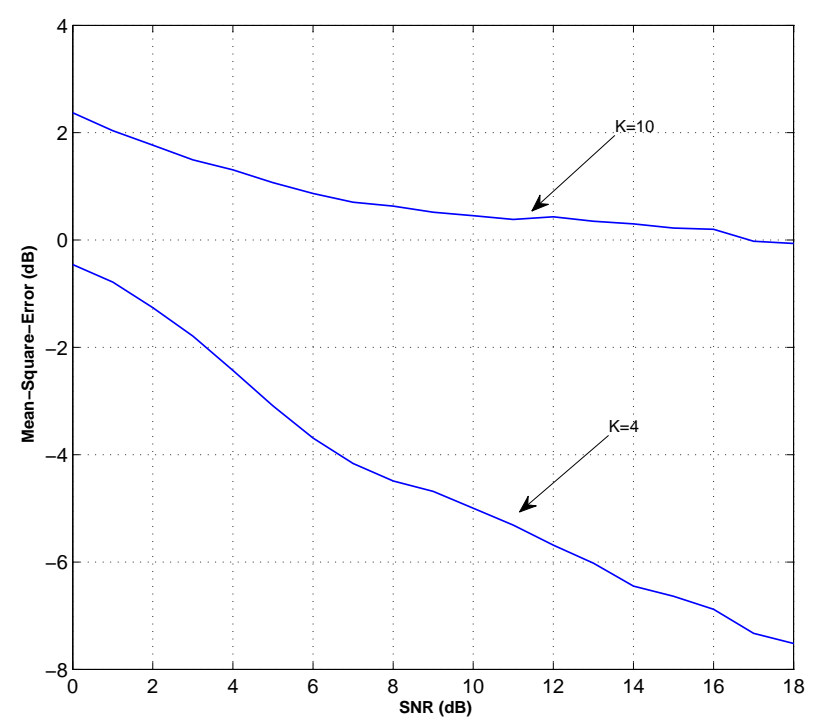

Fig. 2. Mean Squared Error (MSE).

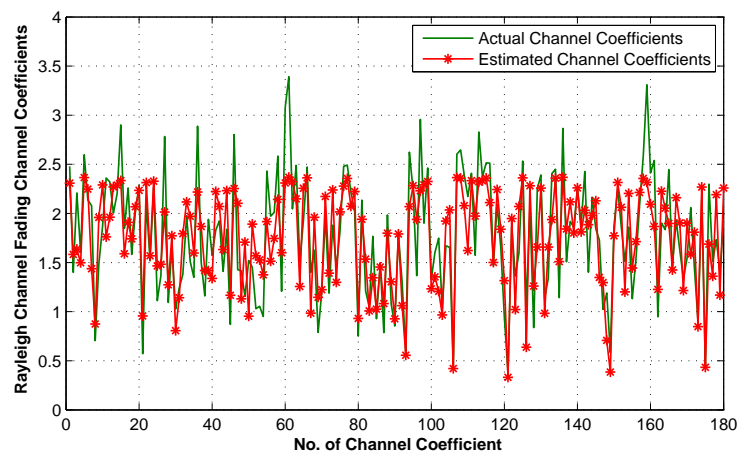

Fig. 3. Channel Coefficients (Rayleigh).

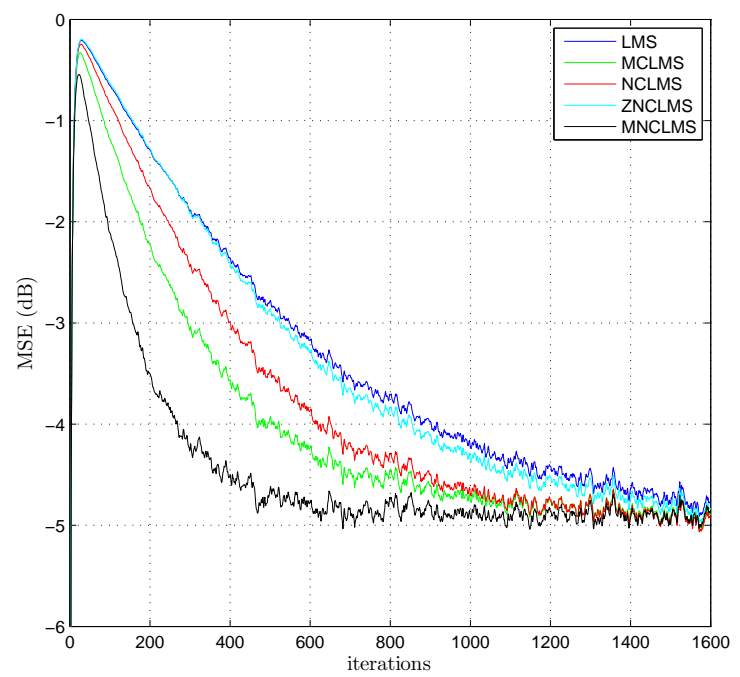

Fig. 4. MSE for competing algorithms for 6 users at SNR value of $10 \mathrm{~dB}$.

Fig. 2 shows MSE of channel estimation for 6 and 12 users for different values of SNR. As shown, performance of channel

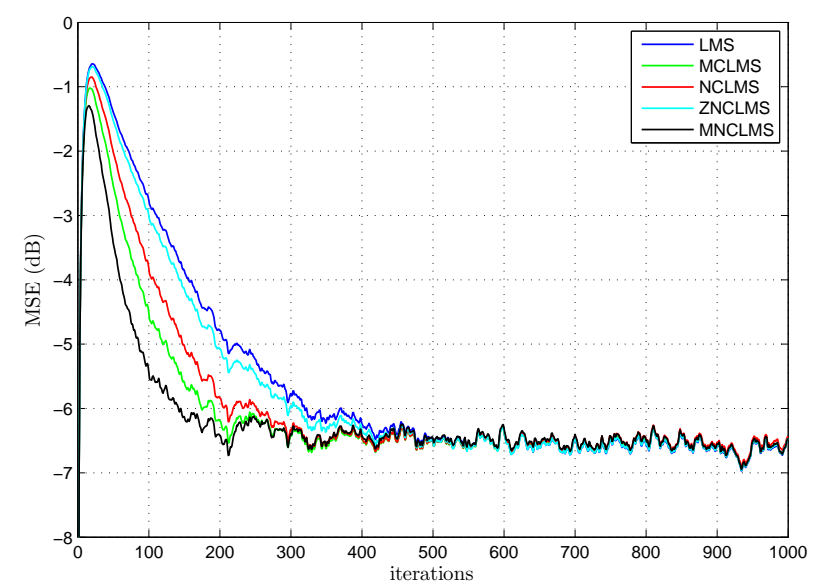

Fig. 5. MSE for competing algorithms for 12 at an SNR of $18 \mathrm{~dB}$.

estimator worsens if more users are added (from 6 to 12). This worsening is the result of MAI at the receiving end. At an SNR value of $17.5 \mathrm{~dB}$, our designed channel estimator attains MSE of $-7.7 \mathrm{~dB}$ for 6 users and MSE of $1.1 \mathrm{~dB}$ for 12 users. Channel estimates of Rayleigh fading channel are compared to that of actual random channel coefficients at $20 \mathrm{~dB}$ SNR in Fig. 3. As observed, our designed estimator tracks random channel coefficients effectively and efficiently.

The proposed estimator is also tested with different constrained algorithms for the linear equalizer [12]. Simulation results in Fig. 4 and 5 show that proposed estimator is working efficiently as MAI and noise constrained algorithm is converging much faster than other constrained algorithms for linear equalization case when used with the proposed estimator.

\section{CONCLUSION}

In this paper, a pilot aided channel estimating technique for MIMO - CDMA systems is presented. This technique is utilizes minimum mean squared error estimation of corrupted information in a flat fading channel along with noise. Simulation results predicts theoretical predictions are strongly validated for different values of SNR and users.

\section{REFERENCES}

[1] M. Salehi and J. Proakis, "Digital communications," McGraw-Hill, New York, 2008.

[2] J. S. Lee and L. E. Miller, CDMA systems engineering handbook. Artech House, Inc., 1998.

[3] E. Dahlman, B. Gudmundson, M. Nilsson, and A. Skold, "Umts/imt2000 based on wideband cdma," Communications Magazine, IEEE, vol. 36, no. 9, pp. 70-80, 1998.

[4] S. Sampei and T. Sunaga, "Rayleigh fading compensation method for 16qam in digital land mobile radio channels," in Vehicular Technology Conference, 1989, IEEE 39th. IEEE, 1989, pp. 640-646.

[5] J. K. Cavers, "An analysis of pilot symbol assisted modulation for rayleigh fading channels [mobile radio]," Vehicular Technology, IEEE Transactions on, vol. 40, no. 4, pp. 686-693, 1991.

[6] A. Aghamohammadi, H. Meyr, and G. Ascheid, "Adaptive synchronization and channel parameter estimation using an extended kalman filter," Communications, IEEE Transactions on, vol. 37, no. 11, pp. 1212-1219, 1989. 
[7] A. D'Andrea, A. Diglio, and U. Mengali, "Symbol-aided channel estimation with nonselective rayleigh fading channels," Vehicular Technology, IEEE Transactions on, vol. 44, no. 1, pp. 41-49, 1995.

[8] M. N. Iqbal and M. Moinuddin, "Pilot-aided rayleigh fading channel estimation using mmse estimator for ds-cdma system," in Multitopic Conference (INMIC), 2011 IEEE 14th International. IEEE, 2011, pp. 347-350.

[9] R. K. Morrow Jr and J. S. Lehnert, "Bit-to-bit error dependence in slotted ds/ssma packet systems with random signature sequences," Communications, IEEE Transactions on, vol. 37, no. 10, pp. 10521061, Oct 1989.

[10] M. Moinuddin, a. U. H. Sheikh, A. Zerguine, and M. Deriche, "A Unified Approach to BER Analysis of Synchronous Downlink CDMA Systems with Random Signature Sequences in Fading Channels with Known Channel Phase," EURASIP Journal on Advances in Signal Processing, vol. 2008, pp. 1-13, 2008. [Online]. Available: http://www.hindawi.com/journals/asp/2008/346465.html

[11] K. Mahmood, S. Asad, M. Moinuddin, A. Zerguine, and S. Paul, "Statistical analysis of multiple access interference in rayleigh fading environment for mimo cdma systems," in Statistical Signal Processing (SSP), 2014 IEEE Workshop on, June 2014, pp. 412-415.

[12] K. Mahmood, S. M. Asad, M. Moinuddin, and S. Paul, "Design of MAI constrained decision feedback equalizer for MIMO CDMA system," 2011 International Conference on Wireless Communications and Signal Processing (WCSP), pp. 1-5, Nov. 2011.

[13] J. Hu, S. Member, and N. C. Beaulieu, "Accurate Simple Closed-Form Approximations to Rayleigh Sum Distributions and Densities," vol. 9, no. 2, pp. 109-111, Feb 2005. 